\title{
Übertragung einiger Sätze aus der Theorie der algebraischen Flächen auf komplexe Mannigfaltigkeiten von zwei komplexen Dimensionen.
}

\author{
Herrn Otto Haupt zum 65. Geburtstag gewidmet.
}

Von Friedrich Hirzebruch in Erlangen, z. Zt. Princeton, N. J.

\section{Einleitung.}

In dieser Arbeit sollen einige Sätze der algebraischen Geometrie über Kurven auf singularitätenfreien algebraischen Flächen mit Hilfe von topologischen Methoden von H. Hopf [12], [13] und E. G. Kundert [15]*) auf kompakte komplexe Mannigfaltigkeiten von zwei komplexen Dimensionen übertragen werden (Definition der komplexen Mannigfaltigkeit z. B. in [11]). Es handelt sich um die Formel für das Geschlecht einer algebraischen Kurve auf einer algebraischen Fläche und um Formeln über die Anzahl der Kurven mit Doppelpunkt eines Kurvenbüschels.

Plückersche Geschlechtsformel ([18], p. 103, (13)).

Eine singularitätenfreie Kurve $K$ der Ordnung $n$ in der komplex-projektiven Ebene hat das Geschlecht $p=\frac{(n-1)(n-2)}{2}$; es ist $2-2 p=-n^{2}+3 n$.

Wir verwenden hier die topologische Geschlechtsdefinition: $K$ ist eine orientierte kompakte Fläche und hat als solche ein (topologisches) Geschlecht $p$ und die Eulersche Charakteristik $2-2 p$.

Hat die Kurve $d$ Doppelpunkte und keine weiteren Singularitäten, so ist ihre Eulersche Charakteristik gleich

$$
2-2 p=-n^{2}+3 n+2 d .
$$

Entsprechende Formeln gelten, wenn andere Singularitäten auftreten. Bei der topologischen Geschlechtsdefinition einer Kurve $K$ mit singulären Punkten ist so vorzugehen:

$K$ wird zu einer orientierten Fläche $K^{*}$, wenn ein singulärer Punkt von $K$, durch den $k$ Zweige hindurchgehen, in ,natürlicher Weise“ als Menge von $k$ verschiedenen Punkten von $K$ aufgefaßt wird. Das (topologische) Geschlecht von $K^{*}$ ist das Geschlecht der Kurve $K$.

Für das Geschlecht einer Kurve auf einer (singularitätenfreien) algebraischen Fläche gelten analoge Formeln ([19], p. 34, 56, 59).

Die Verallgemeinerung der Plückerschen Geschlechtsformeln auf eine kompakte analytische Fläche $H$ (eine komplexe Dimension) in einer kompakten komplexen Mannigfaltigkeit $M$ von zwei komplexen Dimensionen ist der Inhalt des nachstehenden Satzes (vgl. Abschnitt 2.5 dieser Arbeit):

*) Ziffern in [ ] beziehen sich auf das Literaturverzeichnis am Ende der Arbeit. 
Satz I. $H$ sei singularitätenfrei in $M$ eingebettet $\left.{ }^{1}\right)$ und $h$ die durch. $H$ repräsentierte (ganzzahlige) Homologieklasse von M. Ferner sei $c_{1}(M)$ die zur Chernschen charakteristischen zweidimensionalen Cohomologieklasse duale zweidimensionale Homologieklasse, 。 bedeute die Bildung der Schnittzahl und $e(H)$ die Eulersche Charakteristik von H. Dann gilt:

$$
e(H)=-h \circ h+c_{1}(M) \circ h .
$$

Hat $H$ (algebraische) Singularitäten, so kommen zu dem Ausdruck für $e(H)$ gewisse Summanden hinzu, die nur von der lokalen Struktur der Singularitäten abhängen und genau so wie in der algebraischen Geometrie bestimmt werden können.

Die Auflösung der Singularitäten erfolgt mit Hilfe des von H. Hopf [14] für komplexe Mannigfaltigkeiten definierten $\sigma$-Prozesses (Einsetzen einer Trägersphäre, vgl. auch [1], [8], [9]). Man kann allgemein folgende Frage stellen: $M^{(m)}$ sei eine kompakte komplexe Mannigfaltigkeit von $m$ komplexen Dimensionen, $H^{(n)}(n<m)$ eine singularitätenfrei eingebettete kompakte komplexe Mannigfaltigkeit von $n$ komplexen Dimensionen. Welche Beziehungen bestehen zwischen den Chernschen Klassen von $H^{(n)}$ und denen von $M^{(m)}$ ?

Diese Frage hat W. V. D. Hodge im Falle $n=m-1$ durch die ,adjunction formulas" ([10], p. 146) beantwortet, allerdings nur unter zusätzlichen Voraussetzungen über $M^{(m)}$. Man kann diese zusätzlichen Voraussetzungen vermeiden, wie ich in einer anderen Arbeit ausführen möchte (Anwendung von [15]). Der Satz I (für singularitätenfreies $H$ ) ist gerade die adjunction formula für $m=2$ und $n=1^{1 \mathrm{a}}$ ).

Für eine singularitätenfreie algebraische Fläche $M$ wird in der algebraischen Geometrie eine kanonische Klasse definiert, die als zweidimensionale (ganzzahlige) Homologieklasse aufgefaßt werden kann. Sie ist gleich $-c_{1}(M)$ (vgl. [10] und 2. 6, 2. 7 dieser Arbeit).

Aus diesem Grunde ist Satz I die genaue Verallgemeinerung der bekannten Formeln für das Geschlecht einer Kurve auf einer singularitätenfreien algebraischen Fläche.

\section{Kurvenbüschel.}

Ein allgemeines lineares Büschel von Kurven $n$-ter Ordnung mit getrennten Tangenten in der komplexprojektiven Ebene $\mathfrak{B}$ enthält nach Steiner (siehe z. B. Enzyklopädie, III C 4, p. 450, Fußnote 438) $3(n-1)^{2}$ Kurven mit je einem Doppelpunkt.

Ein solches Büschel bestimmt in $\mathfrak{B}$ ein Feld von komplexen Linienelementen. (Dem Punkte $\boldsymbol{P} \in \mathfrak{P}$ wird das Linienelement zugeordnet, das an die durch $\boldsymbol{P}$ gehende Büschelkurve tangential ist.) Dieses Feld hat endlich viele isolierte Singularitäten, nämlich die $3(n-1)^{2}$ Doppelpunkte und die $n^{2}$ Basispunkte. Der Index (vgl. [12]) jeder Singularität ist 1. Die Indexsumme des Feldes ist daher gleich $4 n^{2}-6 n+3$.

H. Hopf ([12], [13]) und E. Kundert [15] haben Schnittflächen in speziellen Faserungen untersucht, ihre Ergebnisse insbesondere auf Felder von komplexen Linienelementen (mit endlich vielen Singularitäten) in komplexen Mannigfaltigkeiten angewandt und die Frage nach den möglichen Indexsummen beantwortet:

Satz von Hopf [12]. Für die komplex-projektive Ebene gilt: Eine ganze Zahl tritt genau dann als Indexsumme eines Feldes komplexer Linienelemente mit endlich vielen Singularitäten auf, wenn sie von der Form $x^{2}+3 x+3$ ist ( $x$ ganz). (Die obige Indexsumme $4 n^{2}-6 n+3$ ist von dieser Form.)

1) Es gibt bekanntlich kompakte komplexe Mannigfaltigkeiten $M$, in denen es keine eingebettete kompakte analytische Fläche $H$ gibt.

1a) Vgl. Zusatz bei der Korrektur 1 am SchluB der Arbeit. 
Wir beweisen in dieser Arbeit einen Satz (vgl. 3. 3), der eine Anwendung der Ergebnisse von H. Hopf und E. Kundert ist und gewisse Sätze der algebraischen Geometrie über lineare Kurvenbüschel verallgemeinert (die Invariante von Zeuthen-Segre spielt hier eine Rolle). Er lautet:

Satz II. $M$ sei eine kompakte komplexe Mannigfaltigkeit von zwei komplexen Dimensionen, $F$ eine meromorphe Funktion in $M$. Die an den Niveauflächen (eine komplexe Dimension) von $\boldsymbol{F}$ tangentialen komplexen Linienelemente bilden ein Feld mit endlich vielen Singularitäten. Die Indexsumme dieses Feldes ist

$$
(2 h-w) \circ(2 h-w)-(2 h-w) \circ c_{1}(M)+c_{2}(M),
$$

wo h die gemeinsame zweidimensionale Homologieklasse der Niveauflächen von $F$ ist, w eine durch $F$ bestimmte zweidimensionale Homologieklasse, die Verzweigung von $F$, und $c_{2}(M)$ die Eulersche Charakteristik von $M$ ist $\left.^{1 \mathrm{~b}}\right)$.

Über die in dieser Arbeit verwendeten Begriffe und Sätze aus der Theorie der gefaserten Räume kann man sich in dem Lehrbuch von Steenrod orientieren [17].

Die in Abschnitt 1 zusammengestellten Hilfssätze sind nicht in der größtmöglichen Allgemeinheit formuliert.

Der Satz I kann auch für fast-komplexe Mannigfaltigkeiten [5] formuliert und bewiesen werden. Wir gehen aber nicht darauf ein.

\section{Zusammenstellung von Hilfssätzen.}

1. 1. $M$ sei eine kompakte komplexe Mannigfaltigkeit von zwei komplexen Dimensionen. $M$ ist durch die komplexe Struktur in natürlicher Weise orientiert $\left.{ }^{2}\right)$. Ein kontravarianter Vektor $\mathfrak{v}$ in $P, P \in M$, wird in bezug auf ein in einer Umgebung von $P$ definiertes zulässiges Koordinatensystem $\left(z_{1}, z_{2}\right)$ von $M$ durch komplexe Koordinaten $\zeta^{1}, \zeta^{2}$ gegeben. Zwei von Null verschiedene kontravariante Vektoren $\mathfrak{v}, \mathfrak{w}$ in $P$ definieren dieselbe kontravariante Richtung in $P$, wenn es ein reelles positives $r$ mit $\mathfrak{v}=r \mathfrak{w}$ gibt. $R(M)$ sei die Mannigfaltigkeit aller kontravarianten Richtungen in $M$. Die Richtungen in einem festen Punkte $P$ von $M$ bilden eine $S^{3}$. Die Mannigfaltigkeit $R(M)$ ist gefasert:

$$
R(M) / S^{3}=M .
$$

Von Null verschiedene kontravariante Vektoren $\mathfrak{v}, \mathfrak{w}$ in $P$ definieren dasselbe komplexe Linienelement in $P$, wenn es eine komplexe Zahl $a$ mit $\mathfrak{v}=a$ w gibt. $L(M)$ sei die Mannigfaltigkeit der komplexen Linienelemente von $M$. Die komplexen Linienelemente in einem festen Punkte $P$ von $M$ bilden eine $S^{2}$ (komplex-projektive Gerade). $L(M)$ ist gefasert:

$$
L(M) / S^{2}=M \text {. }
$$

Jede kontravariante Richtung $\mathfrak{r}$ bestimmt ein komplexes Linienelement $\tau(\mathfrak{r})$. Dies $\tau$ ist eine Faserabbildung von $R(M)$ auf $L(M)$ :

$$
R(M) / S^{1}=L(M)(R(M) \rightarrow L(M) \rightarrow M) .
$$

Analog zu (1) gilt: Die Mannigfaltigkeit $R^{*}(M)$ aller kovarianten Richtungen in $M$ ist gefasert:

$$
R^{*}(M) / S^{3}=M
$$

1b) Vgl. Zusatz bei der Korrektur 2 am Schlu B der Arbeit.

2) Vgl. [11]. Wir beschränken uns in dieser Arbeit auf kompakte komplexe Mannigfaltigkeiten $\boldsymbol{M}$ von zwei komplexen Dimensionen.

Bereitgestellt von | Max-Planck-Gesellschaft - WIB6417 
Jede kovariante Richtung $\mathfrak{r}^{*}$ in $P$ bestimmt ein komplexes Linienelement $\tau^{*}\left(\mathfrak{r}^{*}\right)$ : Wird $\mathfrak{r}^{*}$ in bezug auf ein lokales Koordinatensystem durch Koordinaten $a_{1}, a_{2}$ gegeben, so ist $\tau^{*}\left(\mathrm{r}^{*}\right)$ das durch die Gleichung $a_{1} \zeta^{1}+a_{2} \zeta^{2}=0$ definierte komplexe Linienelement. $\tau^{*}$ ist eine Faserabbildung von $R^{*}(M)$ auf $L(M)$ :

$$
R^{*}(M) / S^{1}=L(M)\left(R^{*}(M) \rightarrow L(M) \rightarrow M\right) .
$$

Wenn im folgenden nur von Richtung gesprochen wird, ist stets kontravariante Richtung gemeint.

Man kann in $M$ stets eine hermitesche Metrik einführen. Für die Faserungen (1) und $\left(1^{*}\right)$ kann die affine unitäre Gruppe, für (2) die projektive unitäre Gruppe, für (3) und $\left(3^{*}\right)$ die eigentliche Drehgruppe der Ebene als Strukturgruppe verwendet werden. Vgl. zu 1. 1 Hirsch [7], p. 128.

1. 2. $\mathrm{Zu}$ diesem Abschnitt siehe [13]; vgl. auch [6], [7], [15]. $M$ ist ein endliches Polyeder. $M$ werde so fein trianguliert, daß die Faserungen (1), (1*) und damit auch (2) über einer geeigneten Umgebung jedes Simplexes trivial sind. Es gibt kontravariante Richtungsfelder in $M$ mit endlich vielen isolierten Singularitäten. Die Faserung (1) und damit auch (2) besitzen daher Schnittflächen über dem 3-dimensionalen Gerüst ${ }^{3}$ ) $M^{3}$ von $M$. Zu jeder Schnittfläche $f$ der Faserung (2) über $M^{3}$ gehört ein 4-dimensionaler Hindernis-Cozyklus ${ }^{4}$ ) $\Gamma_{f}$, der durch eine ganze Zahl, die Indexsumme von $f$, gegeben werden kann. Der Wert von $\Gamma_{f}$ auf einem orientierten Simplex $x^{4}$ ist gleich der ganzen Zahl, die die durch $f$ bestimmte Abbildungsklasse der $S^{3}$ (= orientierter Rand von $x^{4}$ ) in die $S^{2}$ charakterisiert $\left(\pi_{3}\left(S^{2}\right)\right.$ unendlich zyklisch). Für zwei Schnittflächen $f, g$ von (2) über $M^{3}$ ist ein 2-dimensionaler Differenzen-Cozyklus $\alpha(f, g)$ - das Hindernis, das sich der Deformation von $f$ in $g$ auf $M^{2}$ entgegenstellt - definiert. H. Hopf hat einen 2-dimensionalen Schnitt-Cozyklus $\omega(f, g)$ - das Hindernis, das sich der Trennung von $f$ und $g$ auf $M^{2}$ entgegenstellt - definiert. Andeutung der Definition von $\omega(f, g)$ :

$f, g$ lassen sich durch homotope Änderung über $M^{1}$ trennen, d. h es ist $f(P) \neq g(P)$ für $P \in M^{1}$. Für ein Simplex $x^{2}$ lassen sich $f, g$ auffassen als singuläre 2-dimensionale Ketten im kartesischen Produkt $x^{2} \times S^{2}$, deren ganzzahlige Schnittzahl definiert ist. Diese Schnittzahl ist gleich dem Wert von $\omega(f, g)$ auf $x^{2}$.

Die Cohomologieklassen von $\alpha(f, g)$ und $\omega(f, g)$ ändern sich nicht, wenn man $f, g$ homotop abändert. $\alpha(f, g)$ ist cohomolog Null genau dann, wenn $f, g$ auf $M^{2}$ homotop sind.

$\bar{f}$ sei die Schnittfläche der Faserung (2), die jedem Punkt $P$ von $M^{3}$ das zu $f(P)$ in bezug auf eine feste hermitesche Metrik unitär-orthogonale komplexe Linienelemen zuordnet. Die Homotopieklasse von $\bar{f}$ auf $M^{3}$ hängt nicht von der Wahl der hermiteschen Metrik ab.

Es gelten folgende Formeln $(\alpha, \omega, \Gamma$ sollen von nun an die entsprechenden Cohomologieklassen bezeichnen):

$$
\begin{aligned}
& \alpha(f, g)=-\alpha(g, f) \\
& \alpha(f, g)+\alpha(g, h)=\alpha(f, h) \\
& \omega(f, g)=\omega(g, f) \\
& \alpha(f, \bar{g})=\omega(f, g) \\
& \Gamma_{f}-\Gamma_{g}=\alpha(f, g) \cdot \omega(f, g) \text { (Cupprodukt!), } \\
& \alpha(f, g)+\alpha(f, g)=-\omega(g, g) \quad(\text { folgt aus (4) bis (7)), } \\
& \alpha(f, h)+\alpha(g, h)-\omega(f, g)=-\omega(h, h) \\
& \Gamma_{f}=\alpha(f, g) \cdot \alpha(f, g)+\alpha(f, g) \cdot \omega(g, g)+\Gamma_{g} .
\end{aligned}
$$

3) $M^{j}(j=1, \ldots, 4)$ bezeichnet das $j$-dimensionale Gerüst von $M$.

4) Alle in dieser Arbeit vorkommenden Homologie- und Cohomologiegruppen haben den Ring der ganzen Zahlen als Koeffizientenbereich. 
$s$ sei eine Schnittfläche der Faserung (2), die sich vermöge (3) aus einem Richtungsfeld mit endlich vielen Singularitäten von $M$ ergibt. Eine solche Schnittfläche werde Standard-Schnittfläche genannt. Zwei Standard-Schnittflächen der Faserung (2) über $M^{3}$ sind stets auf $M^{2}$ homotop.

Entsprechend werde eine Schnittfläche $s^{*}$ der Faserung (2), die sich vermöge $\left(3^{*}\right)$ aus einem kovarianten Richtungsfeld mit endlich vielen Singularitäten ergibt, als *-Standardschnittfläche bezeichnet. Zwei ${ }^{*}$-Standardschnittflächen von (2) über $M^{\mathbf{3}}$ sind auf $M^{2}$ homotop.

$\gamma_{1}(M)$ sei die 2-dimensionale Chernsche Cohomologieklasse ([4]) von $M$, ferner $\gamma_{2}(M)$ die 4-dimensionale Chernsche Cohomologieklasse von $M$, schließlich $c_{1}(M)$ die zu $\gamma_{1}(M)$ duale Homologieklasse, $c_{2}(M)$ die Eulersche Charakteristik von $M .\left(, c_{2}(M) \cdot\right.$ Punkt" ist dual zu $\left.\gamma_{2}(M)\right)^{4}$ ).

Nach Kundert [15] gilt für eine Standardschnittfläche $s$

$$
\omega(s, s)=\gamma_{1}(M) \text {. }
$$

Für $s$ ist natürlich

$$
\Gamma_{s}=\gamma_{2}(M) .
$$

Aus (10) ergibt sich durch Spezialisierung

$$
\Gamma_{f}=\alpha(f, s) \cdot \alpha(f, s)+\alpha(f, s) \cdot \gamma_{1}(M)+\gamma_{2}(M) .
$$

Zur Faserung (1*) gehören Chernsche Klassen $\gamma_{1}^{*}, \gamma_{2}^{*}$ (2- bzw. 4-dimensionale Cohomologieklasse von $M$ ). Es ist

$$
\gamma_{1}^{*}=-\gamma_{1}, \quad \gamma_{2}^{*}=\gamma_{2} \quad \text { (vgl. [10], p. 141). }
$$

1. 3. Eine Schnittfläche $f$ der Faserung (2) über $M^{3}$ ist eine stetige Abbildung von $M^{3}$ in $L(M)$. Wegen 1.1, (3) und (3*) definiert daher $f$ Faserungen:

$$
E_{f} / S^{1}=M^{3}
$$

und

$$
E_{t}^{*} / S^{1}=M^{3},
$$

wo $E_{f}=\tau^{-1} f\left(M^{3}\right)$ und $E_{f}^{*}=\tau^{*-1} f\left(M^{3}\right)$ ist.

Die zu $P \in M^{3}$ gehörige Faser $S^{1}$ der Faserung (13) besteht aus der $S^{1}$ der Richtungen in $P$, die das komplexe Linienelement $f(P)$ bestimmen. Entsprechend für $\left(13^{*}\right)$. Zur Faserung (13) bzw. (13*) gehört eine 2-dimensionale Chernsche Klasse (1. Hindernis) $\gamma(f)$ bzw. $\gamma^{*}(f)$. Es gilt

$$
\gamma(f)=\alpha(s, f),
$$

wo $s$ irgendeine Standardschnittfläche ist.

Bewveis. Man kann ohne Einschränkung der Allgemeinheit annehmen, daß $s, f$ auf $M^{1}$ übereinstimmen. Für eine Umgebung des Simplexes $x^{2}$ werde die Faserung (2) über $x^{2}$ so als kartesisches Produkt $x^{2} \times S^{2}$ dargestellt, da $\beta f$ in die konstante Abbildung von $x^{2}$ auf einen fes ten Punkt $p_{0}$ von $S^{2}$ übergeht. $s$ gehe aus der Schnittfläche $s_{1}$ der Faserung (1) hervor. Der Wert des durch $s, f$ gegebenen Cozyklus der Klasse $\alpha(s, f)$ auf $x^{2}$ ist das durch die folg ende Abbildung gegebene Element $A$ der Homotopiegruppe $\pi_{2}\left(S^{2}\right)$ : Das orientierte Simplex $x^{2}$ wird durch $s$ in $S^{2}$ abgebildet, der Rand von $x^{2}$ geht in $p_{0}$ über.

Man hat die Hopfsche Faserung $S^{\mathbf{3}} / S^{1}=S^{2}$ mit der Projektionsabbildung $\tau$ (vgl. 1. 1, (3)). Durch die exakte Sequenz der Homotopiegruppen erhält man in bekannter Weise Isomorphismen von $\pi_{2}\left(S^{2}\right)$ auf die relative Homotopiegruppe $\pi_{2}\left(S^{3}, \tau^{-1}\left(p_{0}\right)\right)$ und auf $\pi_{1}\left(S^{1}\right)$. Das Bild von $A$ bei dem-Isomorphismus auf $\pi_{2}\left(S^{3}, \tau^{-1}\left(p_{0}\right)\right)$ wird durch die 
Abbildung $s_{1}$ von $x^{2}$ in $S^{3}$ repräsentiert. Die Abbildung $s_{1}$ auf dem Rande von $x^{2}$ repräsentiert das Bild $A^{\prime}$ von $A$ bei dem Isomorphismus von $\pi_{2}\left(S^{2}\right)$ auf $\pi_{1}\left(S^{1}\right)$. Es ist aber $A^{\prime}$ gerade der Wert des durch $s_{1}$ definierten Cozyklus der Klasse $\gamma(f)$ auf $x^{2}$.

Damit ist (14) bewiesen. Entsprechend gilt

$$
\gamma^{*}(f)=\alpha\left(s^{*}, f\right),
$$

wo $s^{*}$ irgendeine *-Standardschnittfläche ist (vgl. 1. 2).

Es folgt

$$
\gamma(f)-\gamma^{*}(f)=\alpha\left(s, s^{*}\right) .
$$

$\alpha\left(s, s^{*}\right)$ ist unabhängig von der Auswahl der Standardschnittfläche $s$ und der *-Standardschnittfläche $s^{*}$.

Weiter gilt:

$$
\begin{gathered}
\alpha\left(s, s^{*}\right)=\gamma_{1}(M), \\
\gamma(f)=\gamma^{*}(f)+\gamma_{1}(M) .
\end{gathered}
$$

Besveis. In $M$ sei eine hermitesche Metrik eingeführt. $s_{1}$ sei eine Schnittfläche von (1) über $M^{3}$, also ein kontravariantes Richtungsfeld. $s_{1}^{*}$ sei das in bezug auf die hermitesche Metrik zu $s_{1}$ gehörige kovariante Richtungsfeld. $s_{1}^{*}$ ist eine Schnittfläche von (1*) über $M^{3}$. Wir betrachten die Schnittflächen $s=\tau s_{1}$ und $s^{*}=\tau^{*} s_{1}^{*}$ über $M^{3}$ (siehe 1. 1, (3) und $\left.\left(3^{*}\right)\right)$. Wie leicht zu sehen, ist für jeden Punkt $P \in M^{3}$

$$
s(P) \neq s^{*}(P) \text {. }
$$

Daraus ergibt sich: $s^{*}$ ist homotop zu $\bar{s}$ (siehe 1.2) und damit

$$
\alpha\left(s, s^{*}\right)=\alpha(s, \bar{s})=\omega(s, s)=\gamma_{1}(M)
$$

mittels 1. 2, (7) und (11). Aus (15) folgt dann (16a).

\section{Verallgemeinerung der Plückerschen Geschlechtsformel.}

2. 1. $M$ sei eine kompakte komplexe Mannigfaltigkeit von zwei komplexen Dimensionen, $H$ eine in $M$ singularitätenfrei eingebettete analytische Fläche, die kompakt und zusammenhängend sei $^{1}$ ) ( $H$ ist eine abgeschlossene zusammenhängende Punktmenge von $M$; für $P \in H$ gilt: Die Fläche $H$ wird in einer Umgebung von $P$ in bezug auf ein geeignetes lokales Koordinatensystem $\left(z_{1}, z_{2}\right)$ durch $z_{1}=0$ gegeben). $H$ ist eine kompakte orientierte Fläche, die Orientierung wird durch die komplexe Struktur in bekannter Weise bestimmt. $g(H)$ sei ihr Geschlecht, $e(H)$ ihre Eulersche Charakteristik. Es ist $e(H)=2-2 g(H)$.

In $M$ werde eine Hermitesche Metrik eingeführt.

2. 1. 1. Für $P \in H$ bilden die Richtungen in $P$, die tangential zu $H$ sind, eine $S^{1}$. Die zu $H$ tangentiellen Richtungen von $M$ bilden eine 3-dimensionale Mannigfaltigkeit $E_{T}(H)$, die in die Sphären $S^{1}$ der Tangentialrichtungen der Punkte von $H$ gefasert ist:

$$
E_{T}(H) / S^{1}=H \text {. }
$$

$H$, die Fasern und damit $E_{T}(H)$ sind in natürlicher Weise orientiert. Strukturgruppe ist die Gruppe $S^{1}$ der eigentlichen Drehungen in der Ebene. Zur Faserung (17) gehört eine 2-dimensionale Chernsche Cohomologieklasse (1. Hindernis) von $H$, die durch eine ganze Zahl $c_{T}(H)$, nämlich die Indexsumme einer Schnittfläche mit endlich vielen Singularitäten, gegeben werden kann $\left(c_{T}(H)\right.$ ist eine Seifertsche Invariante der 3-dimensionalen gefaserten Mannigfaltigkeit $\left.E_{T}(H),[16]\right) . c_{T}(H)$ hängt nicht von der speziellen Schnittfläche ab. Es gilt $c_{r}(H)=e(H)$. 
2. 1. 2. Für $P \in H$ bilden die Richtungen in $P$, die unitär-orthogonal zu $H$ sind, eine $S^{1}$. Die zu $H$ unitär-orthogonalen (normalen) Richtungen von $M$ bilden eine 3-dimensionale Mannigfaltigkeit $E_{N}(H)$, die in die Sphären $S^{1}$ der Normalrichtungen der Punkte von $H$ gefasert ist:

$$
E_{N}(H) / S^{1}=H .
$$

$H$ ist durch die komplexe Struktur orientiert. $S_{P}^{1}$ sei die zum Punkte $P$ von $H$ gehörige Faser; sie ist so zu orientieren, daß durch die Orientierung von $H$ und die von $S_{P}^{1}$ die natürliche Orientierung von $M$ aufgespannt wird. Zur Faserung (18) gehört eine 2-dimensionale Chernsche Cohomologieklasse von $H$ (1. Hindernis), die durch eine ganze Zahl $c_{N}(H)$, nämlich die Indexsumme einer Schnittfläche mit endlich vielen Singularitäten gegeben werden kann. $c_{N}(H)$ hängt nicht von der speziellen Schnittfläche ab. $h$ sei die durch $H$ repräsentierte 2-dimensionale Homologieklasse von $M$. Es gilt (o bedeutet wieder Schnittzahlbildung)

$$
\left.c_{N}(H)=h \circ h^{5}\right) .
$$

Beweis. $P$ sei ein Punkt von $H$. Es gibt ein lokales Koordinatensystem $(u, v)$ von $M$ mit $P=(0,0)$, so da $\beta H$ gegeben wird durch $u=0$. In einer geeigneten Umgebung $U$ von $P$ kann man in bezug auf dieses Koordinatensystem die folgenden Konstruktionsschritte durchführen. Jeder Punkt $Q \in U \cap H(Q=(0, v))$ wird mit dem Punkt $(v, v)$ geradlinig verbunden. Der Vektor $[\overrightarrow{(0, v),(v, v)}]$ ist, falls $v \neq 0$, d. h. $Q \neq P$ ist, nicht 0 und nicht tangential zu $H$. Ferner sei $s(Q)(Q \neq P)$ die zu $H$ in $Q$ unitär-orthogonale Richtung, die durch die zu $H$ unitär-orthogonale Komponente dieses Vektors gegeben wird. $s(Q)$ ist eine ,lokale" Schnittfläche der Faserung (18). Sie ist in $U \cap H$ definiert und hat in $P$ eine isolierte Singularität mit dem Index 1.

Jeder Punkt $Q \in U \cap H, Q=(0, v)$, werde nun mit dem Punkt $(\bar{v}, v)$ geradlinig verbunden. Der Vektor $[\overrightarrow{(0, v),(\vec{v}, v)]}$ ist, falls $v \neq 0$, d. h. $Q \neq P$ ist, nicht Null und nicht tangential zu $H$. Man erhält jetzt eine „lokale“ Schnittfläche der Faserung (18), die in $U \cap H$ definiert ist und in $P$ eine isolierte Singularität mit dem Index -1 hat.

In geeigneten $H$-Umgebungen endlich vieler Punkte $P_{i}$ von $H\left(i=1, \ldots,\left|c_{N}(H)\right|\right)$ werden nun lokale Schnittflächen der Faserung (18) mit dem Index 1 bzw. -1 so angebracht, daß die Indexsumme gleich $c_{N}(H)$ ist. Diese lokalen Schnittflächen besitzen nach bekannten Sätzen eine gemeinsame Erweiterung $s$, wo $s$ eine Schnittfläche ist, die nur die Singularitäten $P_{i}$ hat. Aus dieser Schnittfläche $s$ kann man nun eine 2-dimensionale kompakte orientierte in $M$ liegende Fläche $F_{s}$ konstruieren, die $H$ in den Punkten $P_{i}$ mit der Schnittzahl 1 bzw. -1 schneidet. (Man repräsentiert das Richtungsfeld $s$ durch ein Vektorfeld $\mathfrak{v}$, das genau in den $\boldsymbol{P}_{\boldsymbol{i}}$ Nullstellen hat. Der Vektor $\mathfrak{v}$ im Punkte $\boldsymbol{P} \in M$ werde gegeben durch eine kleine Verrückung von $P$ in einen benachbarten Punkt, den „Endpunkt des Vektors". Vgl. Alexandroff-Hopf, Topologie, p. 549. $F_{s}$ ist die Fläche der Endpunkte dieserVektoren.) $F_{s}$ ist zu $H$ homolog. Die Schnittzahl von $F_{s}$ mit $H$ ist gleich $c_{N}(H)$.

2. 2. Satz. $M$ und $H$ seien wie in 2.1 definiert. Die Eulersche Charakteristik $e(H)$ hängt nur von der Homologieklasse $h$ von $H$ ab und zwar gilt

$$
e(H)=-h \circ h+\gamma_{1} h,
$$

wo $\gamma_{1}$ die 2-dimensionale Chernsche Klasse von $M$ ( von $\gamma_{1}$ mit $h\left(\gamma_{1}\right.$ angewandt auf $\left.h\right)$ und $\circ$ die Bildung der Schittzahl bedeutet. Bezeichnet ferner $c_{1}$ die $z u \quad \gamma_{1}$ duale 2-dimensionale Homologieklasse, so gilt

$$
e(H)=-h \circ h+c_{1} \circ h .
$$

5) Diesen Satz kenne ich durch Herrn H. Hopf. 
Beweis. $M$ kann so trianguliert werden, daß $H$ ein Teilkomplex ist ${ }^{6}$ ) Die Triangulation sei so fein wie in 1.2 und $s_{1}$ eine Schnittfläche der Faserung (17) mit endlich vielen Singularitäten $O_{i} \in H$. Ferner sei $s_{2}$ eine Schnittfläche von (18) mit Singularitäten $P_{i} \in H$. Es sei stets $O_{i} \neq P_{j}$. Die $O_{i}, P_{j}$ sollen im Innern von 2 -Simplexen von $H$ liegen, in jedem 2-Simplex liege höchstens eine Singularität. $\left(s_{1}, s_{2}\right)$ definiert ein 2-Feld auf $H$, das auf das 2-Gerüst von $M$ erweitert werden kann. $\gamma_{1} h$ ist die Singularitätensumme von $\left(s_{1}, s_{2}\right)$ auf $H$; das bedeutet: Der Rand $x^{2}$ des orientierten 2-Simplexes $x^{2}$ von $H$ wird durch $\left(s_{1}, s_{2}\right)$ in $U_{2}$ (Raum der unitär-orthogonalen 2-Beine = unitäre Gruppe) abgebildet. Diese Abbildung repräsentiert ein Element der unendlich-zyklischen Gruppe $\pi_{1}\left(U_{2}\right)$. Jedem Simplex $x^{2}$ von $H$ ist so eine ganze Zahl zugeordnet, die mit $\sigma_{1}\left(x^{2}\right)$ bezeichnet werde. Die Summe dieser Zahlen für alle 2-Simplexe von $H$ ist $\gamma_{1} h$. Wenn $s_{1}$ über $x^{2}$ keine Singularität hat, so ist, wie leicht einzusehen, $\sigma_{1}\left(x^{2}\right)$ gleich dem Index von $s_{2}$. Entsprechend für $s_{2}$. Es folgt:

$$
\begin{aligned}
& \gamma_{1} h=\text { Indexsumme von } s_{1}+\text { Indexsumme von } s_{2} \text {, } \\
& \gamma_{1} h=e(H)+h \circ h \text {. }
\end{aligned}
$$

2. 3. $M$ sei wie bisher eine komplexe Mannigfaltigkeit von zwei komplexen Dimensionen, $Q$ ein Punkt von $M$. Wir verwenden den folgenden, aus der algebraischen Geometrie bekannten und von H. Hopf ([14], vgl. auch [8], p. 85) für komplexe Mannigfaltigkeiten betrachteten Einsetzungsprozeß ( $\sigma$-Prozeß).

Mit $\sigma_{Q} M$ werde die komplexe Mannigfaltigkeit bezeichnet, die aus $M$ durch Herausstechen von $Q$ und darauf folgendes analytisches Einsetzen von $T_{Q}$ entsteht. $T_{Q}$ bezeichnet dabei die Sphäre $S^{2}$ (= komplex-projektive Gerade = Riemannsche Zahlenkugel) der komplexen Linienelemente in $Q$, die eine kompakte analytische Fläche vom Geschlecht Null ist und singularitätenfrei in $\sigma_{Q} M$ liegt. Es gibt eine analytische Abbildung $t_{Q}$ von $\sigma_{Q}$ $M$ auf $M$ mit folgenden Eigenschaften:

1. $t_{Q}$ bildet $T_{Q}$ auf $Q$ ab.

2. $t_{Q}$ bildet $\sigma_{Q} M-T_{Q}$ eineindeutig auf $M-Q \mathrm{ab}$.

3. Wenn $z_{1}, z_{2}$ lokale Koordinaten in einer Umgebung von $Q$ sind, so gibt es in $\sigma_{Q} M$ lokale Koordinatensysteme $\left(z_{1}^{\prime}, z_{2}^{\prime}\right),\left(z_{1}^{\prime \prime}, z_{2}^{\prime \prime}\right)$, die eine Umgebung von $T_{Q}$ überdecken und für die $t_{Q}$ gegeben wird durch

$$
\begin{array}{ll}
z_{1}=z_{1}^{\prime}, & z_{1}=z_{1}^{\prime \prime} z_{2}^{\prime \prime}, \\
z_{2}=z_{1}^{\prime} z_{2}^{\prime}, & z_{2}=z_{2}^{\prime \prime} .
\end{array}
$$

Für die durch $T_{Q}$ repräsentierte Homologieklasse $\tau_{Q}$ von $\sigma_{Q} M$ gilt

$$
\tau_{Q} \circ \tau_{Q}=-1
$$

Jede $j$-dimensionale $(j<4)$ Homologieklasse $h$ von $M$ läßt sich durch einen Zyklus repräsentieren, $\operatorname{der} Q$ nicht trifft und deshalb sofort als Zyklus von $\sigma_{Q} M$ aufgefaßt werden kann und eine Homologieklasse $t_{Q}^{*} h$ von $\sigma_{Q} M$ repräsentiert. $t_{Q}^{*}$ bildet den Schnittring von $M$ isomorph in den von $\sigma_{Q} M$ ab (Hopfscher Umkehrungshomomorphismus). Die erste und dritte Homologiegruppe von $M$ und $\sigma_{Q} M$ sind isomorph (Isomorphismus $t_{Q}^{*}$ ). Für die zweite Homologiegruppe gilt

$$
H^{2}\left(\sigma_{Q} M\right)=t_{Q}^{*} H^{2}(M)+\left(\tau_{Q}\right) \quad(\dot{+}=\text { direkte Summe }),
$$

6) Die in dieser Arbeit vorkommenden Triangulierbarkeitsaussagen lassen sich aus den Arbeiten [2], [3] von Cairns mit Hilfe von einigen Zusätzen zu den dortigen Beweisanordnungen entnehmen. 
wo $\left(\tau_{Q}\right)$ die von $\tau_{Q}$ erzeugte unendlich-zyklische Gruppe bezeichnet. Die Schnittmatrix von $\sigma_{Q} M$ wird durch (21) und durch

$$
t_{Q}^{*} h \circ \tau_{Q}=0 \text { für } h \in H^{2}(M)
$$

gegeben.

Für die 2-dimensionalen Chernschen Homologieklassen von $M$ und $\sigma_{Q} M$ gilt, wie sofort zu sehen ist,

$$
c_{1}\left(\sigma_{Q} M\right)=t_{Q}^{*} c_{1}(M)+m \tau_{Q} \quad(m \text { ganze Zahl }) .
$$

Aus

2. 2, (20a) folgt

$$
-\tau_{Q} \circ \tau_{Q}+c_{1}\left(\sigma_{Q} M\right) \circ \tau_{Q}=1-m=2 \text {, d. h. } m=-1 \text {. }
$$

Es gilt also

$$
c_{1}\left(\sigma_{Q} M\right)=t_{Q}^{*} c_{1}(M)-\tau_{Q} .
$$

2. 4. $H$ sei eine kompakte analytische zusammenhängende Fläche in der kompakten komplexen Mannigfaltigkeit $M$ mit einem Doppelpunkt $D$. Sie wird zu einer orientierten topologischen Fläche $H^{*}$, wenn man in natürlicher Weise den Doppelpunkt $D$ von $H$ als Menge von zwei verschiedenen Punkten von $H^{*}$ auffaßt. Unter $e(H)$ soll die Eulersche Charakteristik von $H^{*}$ verstanden werden. $H$ ist eine abgeschlossene Teilmenge von $M$. Für $P \in H$ und $P \neq D$ gibt es eine Umgebung $U$ und ein lokales Koordinatensystem $z_{1}, z_{2}$ in $U$, so daß $H$ in $U$ durch $z_{1}=0$ gegeben wird. Es gibt eine Umgebung $V$ von $D$ und ein lokales Koordinatensystem $v_{1}, v_{2}$ in $V$ so, da $H$ in $V$ durch $v_{1} v_{2}=0$ gegeben wird. In $D$ werde $T_{D}$ eingesetzt (2.3). In $\sigma_{D} M$ liegt jetzt eine singularitätenfreie analytische Fläche $H^{*}$, die $T_{D}$ in zwei Punkten einfach schneidet und außerhalb $T_{D}$ durch $t_{D}$ eineindeutig auf $H$ abgebildet wird. Die Eulersche Charakteristik $e\left(H^{*}\right)$ bestimmt sich jetzt so:

$h, h^{*}$ seien die Homologieklassen von $H, H^{*}$ in $M$ bzw. $\sigma_{D} M$. Hierfür gilt:

$$
h^{*}+2 \tau_{\boldsymbol{D}}=t_{\boldsymbol{D}}^{*} h .
$$

Damit erhält man aus 2. 2, (20a) unter Verwendung von (21) und (23)

$$
\begin{aligned}
e(H)=e\left(H^{*}\right) & =-h^{*} \circ h^{*}+h^{*} \circ c_{1}\left(\sigma_{D} M\right) \\
& =-\left(t_{D}^{*} h-2 \tau_{D}\right) \circ\left[\left(t_{D}^{*} h-2 \tau_{D}\right)-\left(t_{D}^{*} c_{1}(M)-\tau_{D}\right)\right] \\
& =-h \circ h+h \circ c_{1}(M)+2 .
\end{aligned}
$$

Durch einen Doppelpunkt wird also wie in der algebraischen Geometrie die Eulersche Charakteristik um 2 vermehrt, das Geschlecht um 1 vermindert.

Durch wiederholte Anwendung des $\sigma$-Prozesses (Einsetzen von $\sigma$-Bäumen [14]) kann man wie in der algebraischen Geometrie jede beliebige algebraische Singularität einer analytischen Fläche auflösen ([9]), wie wir es am Beispiel des Doppelpunktes durchgeführt haben. Man erhält dann folgendes Ergebnis:

2. 5. Satz. $M$ sei eine kompakte komplexe Mannigfaltigkeit, $H$ eine kompakte analytische Fläche in $M$. Wir lassen zu, daß $H$ (algebraische) Singularitäten hat und daß $H$ reduzibel ist. Wir setzen voraus, daß $H$ keine mehrfachen Bestandteile enthält. $H$ wird zu einer kompakten orientierten topologischen estl. nicht zusammenhängenden Fläche $H^{*}$, wenn man einen singulären Punkt von $H$, durch den $k Z$ weige hindurchgehen, in ,natürlicher" Weise als Menge von $k$ verschiedenen Punkten von $H^{*}$ auffaßt. $H^{*}$ liegt als singularitätenfreie analytische Fläche in einer komplexen Mannigfaltigkeit $M^{*}$, die aus $M$ durch Einsetzen von $\sigma$-Bäumen in die singulären Punkte von $H$ hervorgeht. Unter $e(H)$ werde die Eulersche 
Charakteristik von $\mathrm{H}^{*}$ (=Summe der Charakteristiken der Zusammenhangskomponenten von $\left.H^{*}\right)$ verstanden. Wie in der algebraischen Geometrie kann jeder Singularität $Q$ eine $Z$ ahl $a_{Q}$ zugeordnet werden: $a_{Q}=\Sigma v_{j}\left(v_{j}-1\right)$. Die Summe ist zu erstrecken über die Vielfachheiten von $\mathrm{H}$ in $\mathrm{Q}$ und den „,unendlich benachbarten" Punkten von $Q$, die in naheliegender Weise als Punkte auf in $Q$ eingesetzten $\sigma$-Bäumen zu deuten sind. $a_{Q}$ hängt nur von der lokalen Struktur der Singularität ab. $h$ sei die durch $H$ repräsentierte Homologieklasse von $M, c_{1}$ die 2-dimensionale Chernsche Homologieklasse von M. Dann gilt:

$$
e(H)=-h \circ h+c_{1} \circ h+\Sigma a_{Q} .
$$

Die Summe ist über alle Singularitäten von $H z$ zu erstrecken.

2. 6. Der Satz 2. 5 ist die genaue Verallgemeinerung der Formel über das Geschlecht einer algebraischen Kurve auf einer singularitätenfreien algebraischen Fläche, wenn man die Tatsache beachtet, daß für eine algebraische Fläche $-c_{1}$ die kanonische Klasse der Fläche ist (vgl. [10]).

Für die komplex-projektive Ebene ist die kanonische Klasse gleich

$-3 \cdot$ projektive Gerade $=-$ Chernsche Klasse.

Die Formel in 2.5 besagt dann: Die Charakteristik einer Kurve $n$-ter Ordnung ist gleich

$$
-n^{2}+3 n+\Sigma a_{Q}
$$

Die Übereinstimmung der Chernschen Klasse mit der negativen kanonischen Klasse ergibt sich aus folgendem Satz:

2. 7. Es wird vorausgesetzt, daß es in $M$ zwei unabhängige meromorphe Funktionen $F_{1}, F_{2}$ gibt. Durch die Funktionaldeterminante $\frac{\partial\left(F_{1}, F_{2}\right)}{\partial\left(z_{1}, z_{2}\right)}\left(z_{1}, z_{2}\right.$ beliebige lokale Koordinaten in $M$ ) wird eine Verteilung von Null- und Polstellenflächen mit ganzzahligen Vielfachheiten gegeben (in üblicher Weise: Nullstellenfläche positive, Polstellenfläche negative Vielfachheit). Hierdurch wird ein Zyklus $C$ von $M$ gegeben. Die Homologieklasse von $C$ hängt nicht von der Auswahl von $F_{1}, F_{2}$ ab, wie direkt einzusehen ist. Sie ist gleich $-c_{1}(M)$.

Ein Beweis dieses Satzes soll in 3.6.1 angedeutet werden.

\section{Verallgemeinerung von Sätzen über Kurvenbüschel auf algebraischen Flächen} (Invariante von Zeuthen-Segre).

3.1. $M$ sei eine kompakte komplexe Mannigfaltigkeit $\left.{ }^{2}\right), F$ eine in $M$ definierte nicht konstante meromorphe Funktion. (Es gibt bekanntlich komplexe Mannigfaltigkeiten, in denen keine nicht konstanten meromorphen Funktionen existieren!) Die an die Niveauflächen von $F$ tangentiellen komplexen Linienelemente bilden ein Feld $f$ mit endlich vielen Singularitäten (Schnittfläche der Faserung 1. 1, (2)). Die Indexsumme von $f$ (vgl. 1. 2) soll bestimmt werden.

$H_{a}$ sei die $a$-Stellenfläche von $F$ ( $a$ komplexe Zahl oder $a=\infty ; H_{\infty}$ Polstellenfläche von $F$ ). Ein Punkt $P$ von $M$ heißt (algebraisch-)singulärer Punkt von $F$, wenn $F$ in $P$ eine Unbestimmtheitsstelle oder wenn die durch $P$ gehende $H_{F(P)}$ in $P$ eine Singularität hat (d. h. es gibt kein lokales Koordinatensystem $z_{1}, z_{2}$ mit $P=(0,0)$, in dem $H_{F(P)}$ durch $z_{1}=0$ gegeben wird). Es gibt nur endlich viele singuläre Punkte $S_{i}$ von $F$. Ist $P$ kein singulärer Punkt, so bezeichne $f(P)$ das in $P$ an $H_{F(P)}$ tangentielle komplexe Linienelement. $f$ ist eine Schnittfläche der Faserung 1.1,(2) mit den endlich vielen Singularitäten $S_{i}$. 
$h$ bezeichne die (von $a$ unabhängige) 2-dimensionale Homologieklasse, die durch $H_{a}$ repräsentiert wird. Die einzelnen irreduziblen Komponenten von $H_{a}$ sind dabei mit den entsprechenden positiven Vielfachheiten zu versehen. $D_{a, 1}, \ldots, D_{a, n_{a}}$ seien die irreduziblen Komponenten von $H_{a}$. Die entsprechenden Vielfachheiten seien $v_{a, j}\left(j=1, \ldots, n_{a}\right)$. Sie geben die Ordnung des Verschwindens von $F-a(a \neq \infty)$ bzw. $1 / F$ auf $D_{a, j}$ bzw. $D_{\infty, j}$ an. Wir setzen

$$
W_{a}=\sum_{j}\left(v_{a, j}-1\right) D_{a, j}
$$

Nur für endlich viele $a$ gibt es Komponenten $D_{a, j}$ mit $v_{a, j}>1$.

Nur für endlich viele $a$ ist also $W_{a} \neq 0$. Schließlich sei w die durch die Summe aller $W_{a}$ repräsentierte Homologieklasse von $M$, die kurz als Verzweigung bezeichnet werde.

3. 2. Satz. s sei eine Standardschnittläche der Faserung 1. 1, (2) und $f, h$, w seien wie in 3. 1 definiert. Dann ist $\alpha(s, f)$ die $z u-2 h+w+c_{1}(M)$ duale Cohomologieklasse.

Beweis. Ohne Einschränkung der Allgemeinheit können wir annehmen, daß $W_{\infty}=0$ ist. In $M-H_{\infty}$ ist $F$ regulär. $z_{1}, z_{2}$ seien lokale Kòordinaten von $M$. Dann ist $\left(\partial F / \partial z_{1}\right.$, $\left.\partial F / \partial z_{2}\right)$ ein in $M-H_{\infty}$ definiertes kovariantes Vektorfeld. In $M-H_{\infty}-\Sigma W_{a}-\left\{S_{i}\right\}$ ist $\left(\partial F / \partial z_{1}, \partial F / \partial z_{2}\right) \neq 0$, bestimmt also dort ein kovariantes Richtungsfeld $\mathfrak{r}^{*}(P)$. Es ist $\tau^{*} \mathfrak{r}^{*}(P)=f(P)$ (vgl. 1. 1). $M$ kann so trianguliert werden, daß folgendes gilt $\left.{ }^{6}\right)$ :

1. Die $S_{i}$ sind Nullsimplexe.

2. $H_{\infty}$ und jede $W_{a}$ sind 2-dimensionale Teilkomplexe.

3. Die Triangulation erfüllt die Bedingung von 1.2. Die duale Zellteilung erfüllt die analoge Bedingung: Über einer geeigneten Umgebung jeder Zelle sind (1) und $\left(1^{*}\right)$ trivial.

$M^{j}(j=1, \ldots, 4)$ bezeichne hier das $j$-dimensionale Gerüst der dualen Zellteilung.

$f$ ist eine Schnittfläche der Faserung (2) über $M^{3}$, ebenso $\mathfrak{r}^{*}$ eine Schnittfläche von 1. 3, $\left(13^{*}\right)$ über $M^{1}$. Bezeichnet $\Gamma^{*}(f)$ den 2-dimensionalen Hindernis-Cozyklus von $\mathfrak{r}^{*}$, so ist der Wert von $\Gamma^{*}(f)$ auf einer Zelle $x^{2}$ von $M^{2}$, die $H_{\infty}$ und $\Sigma W_{a}$ nicht schneidet, Null. Ferner gilt:

(*) Der Wert von $\Gamma^{*}(f)$ auf einer Zelle $x^{2}$, die $H_{\infty}$ mit der Schnittzahl 1 schneidet, ist gleich -2 .

Denn in einer Umgebung $U$ jedes nichtsingulären Punktes $P$ von $H_{\infty}$ gilt $1 / F=z_{1}$ in bezug auf geeignete lokale Koordinaten $\left(z_{1}, z_{2}\right)$, und $H_{\infty}$ wird in $U$ durch $z_{1}=0$ gegeben. Man hat daher $\partial F / \partial z_{1}=-1 / z_{1}^{2}, \partial F / \partial z_{2}=0$.

$\left(^{* *}\right)$ Der Wert von $\Gamma^{*}(f)$ auf einer Zelle $x^{2}$, die einen irreduziblen Bestandteil $D_{a, j}$ (Vielfachheit $v_{a, j}>1$ ) von $W_{a}$ mit der Schnittzahl 1 schneidet, ist $v_{a, j}-1$.

Denn in einer Umgebung $U$ jedes nichtsingulären Punktes $P$ von $D_{a, j}$ gilt $F-a=z_{1}^{v_{a, j}}$ in bezug auf geeignete lokale Koordinaten $\left(z_{1}, z_{2}\right)$, und $D_{a, j}$ wird durch $z_{1}=0$ gegeben. Es ist daher $\partial F / \partial z_{1}=v_{a, j} z_{1}^{0} a, j^{-1}, \partial F / \partial z_{2}=0$.

Aus (*) und (**) folgt: Die Cohomologieklasse $\gamma^{*}(f)$ von $\Gamma^{*}(f)$ ist dual zu $-2 h+w$. Aus 1. 3, (14), (16a) erhält man dann die zu beweisende Behauptung.

3. 3. Die Indexsumme der Schnittfläche $f$ ist eine ganze Zahl, die mit $c_{f}$ bezeichnet werde (vgl. 1. 2). Es gilt

$$
c_{f}=(2 h-w) \circ(2 h-w)-(2 h-w) \circ c_{1}(M)+c_{2}(M),
$$

wie sofort aus 1. 2, (10a) und 3. 2 folgt. Wir haben also insgesamt den

Satz. $F$ sei eine in der kompakten komplexen Mannigfaltigkeit $\left.{ }^{2}\right) M$ definierte meromorphe Funktion. h sei die (gemeinsame) Homologieklasse der Niveaustellenflächen von $F$, 
w die Verzweigung (vgl. 3.1) pon $F, c_{1}(M)$ die zur 2-dimensionalen Chernschen Cohomologieklasse duale Homologieklasse von $M, c_{2}(M)$ die Eulersche Charakteristik von M. Dann wird die Indexsumme $c_{f}$ des Feldes $f$ der an die Niveauflächen tangentiellen komplexen Linienelemente durch die Formel (25) gegeben.

3. 4. Von einer allgemeinen meromorphen Funktion $F$ wollen wir sprechen, wenn $F$ folgende Eigenschaften hat:

1. Es ist $W_{a}=0$.

2. $F$ hat $h \circ h$ Unbestimmtheitsstellen. Jede Unbestimmtheitsstelle $P$ ist vom Typus $z_{1} / z_{2}$, d. h. in einer geeigneten Umgebung $U$ von $P$ gibt es ein lokales Koordinatensystem $z_{1}, z_{2}$, in $\operatorname{dem} F\left(z_{1}, z_{2}\right)=z_{1} / z_{2}$ gilt.

3. Jede andere Singularität $P$ von $F$ ist Doppelpunkt von $H_{\boldsymbol{F}(\boldsymbol{P})}(\operatorname{vgl}$. 2. 4).

4. Jede Fläche $H_{a}$ besitzt höchstens einen Doppelpunkt.

Wie sofort zu sehen, hat $f$ in jedem singulären Punkt $P$ den Index 1. Aus 3.3 folgt nun:

Eine allgemeine meromorphe Funktion $F$ besitzt $3 h \circ h-2 h \circ c_{1}(M)+c_{2}(M)$ Niveauflächen mit Doppelpunkt.

3. 5. Zusammenhang mit bekannten Sätzen der algebraischen Geometrie. Als Anwendung des Bisherigen untersuchen wir einige bekannte Aussagen mit den Mitteln von 3.1 bis 3. 4 .

3. 5. 1. $M$ sei zunächst die komplex-projektive Ebene. In diesem Fall gilt:

$$
c_{1}=3 \cdot \text { projektive Gerade, } c_{\mathbf{2}}=3 \text {. }
$$

Der Satz in 3. 4. besagt: Ein allgemeines lineares Büschel von Kurven $n$-ter Ordnung enthält

$$
3 n^{2}-6 n+3=3(n-1)^{2}
$$

Kurven mit Doppelpunkt ${ }^{7}$ ).

Einfachstes Beispiel: Ein Kegelschnittbüschel $(n=2)$ enthält drei Kurven mit Doppelpunkt, nämlich die drei in Geradenpaare zerfallenden Kegelschnitte des Büschels.

Nun sei $M$ eine (singularitätenfreie) algebraische Fläche. Dann gilt ([19], p. 62):

Let $\{C\}$ be an irreducible linear pencil of curves, of genus $\pi$ and degree $n$, and let $\delta$ be the number of curves in $\{C\}$ which are of genus $\pi-1$. Then $I=\delta-n-4 \pi$. It can be shown that this expression is independent of the pencil $\{C\}$.

Hierin ist $I$ die Zeuthen-Segre-Invariante. Diese Aussage der algebraischen Geometrie läßt sich aus 3.4 so herleiten:

Die gemeinsame Homologieklasse der Kurven von $\{C\}$ ist $h$. Nach Definition des Grades einer Kurve auf einer algebraischen Fläche ist $n=h \circ h$. Die Kurven mit Doppelpunkt haben das Geschlecht $\pi-1$ (vgl. 2. 4). $\pi$ ist das (gemeinsame) Geschlecht der übrigen Kurven von $\{C\}$. Die Anzahl der Kurven mit Doppelpunkt ist gleich $\delta$. Es ist dann (vgl. 2. 2, (20a))

Daher folgt

$$
2-2 \pi=-h \circ h+c_{1}(M) \circ h .
$$

$$
\begin{gathered}
I=\delta-n-4 \pi=3 h \circ h-2 h \circ c_{1}(M)+c_{2}(M)-h \circ h+2\left(-h \circ h+c_{1}(M) \circ h-2\right), \\
I=c_{2}(M)-4 .
\end{gathered}
$$

Wir haben noch die bekannte Tatsache ([19], p. 113) mitbewiesen, daß $I+4$ die Eulersche Charakteristik von $M$ ist.

7) Die Kurven eines linearen Büschels sind stets die Niveauflächen einer in der komplex-projektiven Ebene definierten meromorphen Funktion (siehe $[18]_{\&}$ p. 184). 
3. 5. 2. Wir kehren nun zum Ausgangspunkt von 3.1 zurück. Die irreduziblen Komponenten der Niveauflächen von $F$ lassen sich in natürlicher Weise ${ }^{8}$ ) in Äquivalenzklassen einteilen. (Zwei irreduzible Komponenten einer Fläche $H_{a}$, die sich schneiden, gehören dabei stets zur gleichen Äquivalenzklasse.) Die Äquivalenzklassen können in natürlicher Weise ${ }^{8}$ ) als Punkte einer (kompakten) Riemannschen Fläche $\mathfrak{F}$ aufgefaßt werden. Unter dem Geschlecht $\varrho(F)$ von $F$ werde das Geschlecht dieser Fläche $\mathfrak{F}$ verstanden. $Q_{i}$ seien die Unbestimmtheitsstellen von $F$. Ordnet man jedem Punkt $P$ von $M-\left\{Q_{i}\right\}$ die Äquivalenzklasse der durch ihn hindurchgehenden Komponenten von $H_{F(P)}$ zu, so erhält man eine analytische Abbildung $F^{\prime}$ von $M-\left\{Q_{i}\right\}$ auf $\mathfrak{F}$.

Satz. Wenn $F$ eine Unbestimmtheitsstelle besitzt, so ist $\varrho(F)=0$.

(In [19], p. 25 findet sich ein entsprechender Satz über Kurvenbüschel auf algebraischen Flächen.)

Beweis. F kann so als verzweigte analytische Überlagerung der Riemannschen Zahlenkugel $S^{2}$ mit der Überlagerungsabbildung $G$ aufgefaßt werden, daß $F=G F^{\prime}$ ist. $P$ sei eine Unbestimmtheitsstelle von $F$ und es werde $\varrho(F)>0$ angenommen. $S^{3}$ sei der Rand einer geeigneten Umgebung von $P$. Die Hopfsche Invariante $\gamma$ der Abbildung $F \mid S^{3}$ von $S^{3}$ auf $S^{2}$ ist $\neq 0$, nämlich gleich der Schnittzahl von $H_{0}$ und $H_{\infty}$ in $P$ (hierbei bezeichnєt $F \mid S^{3}$ die auf $S^{3}$ beschränkte Abbildung $\left.F\right)$. Die Abbildung $F^{\prime} \mid S^{3}$ von $S^{3}$ auf $\mathfrak{F}$ ist nullhomotop, da $\pi_{3}(\mathfrak{F})=0$ ist. Also wäre auch $F \mid S^{3}$ nullhomotop. Widerspruch zu $\gamma \neq 0$.

3. 5. 3. Wir betrachten jetzt allgemein eine analytische Abbildung $F^{\prime}$ von $M$ auf eine Riemannsche Fläche $\mathfrak{F}$ vom Geschlecht $\varrho$. Ist $H_{a}(a \in \mathfrak{F})$ die $a$-Stellenfläche von $F^{\prime}$, so schneiden sich $H_{a}, H_{b}$ nicht für $a \neq b$. Analog zu 3.1. sei $h\left(F^{\prime}\right)$ die gemeinsame Homologieklasse der $H_{a}$. Ebenso wie in 3. 1. ist eine Verzweigungs-Homologieklasse $w\left(F^{\prime}\right)$ definiert. $f$ sei das durch die $H_{a}$ definierte Feld von komplexen Linienelementen. Dann ist

$$
\alpha(s, f) \text { dual zu }(2 \varrho-2) h\left(F^{\prime}\right)+w\left(F^{\prime}\right)+c_{1}(M) .
$$

Beweis. $G$ sei eine analytische verzweigte $n$-blättrige Überlagerungsabbildung von $\mathfrak{F}$ auf die Riemannsche Zahlenkugel $S^{2}$. Die Anzahl der Verzweigungspunkte (mit Vielfachheiten) sei $d$ (ein $k$-blättriger Verzweigungspunkt hat die Vielfachheit $k-1$ ). Wir wenden 3. 2 mit $h=h(F)$, w $=w(F)$ auf die meromorphe Funktion $F=G F^{\prime}$ an und erhalten:

$\alpha(s, f)$ ist dual $\mathrm{zu}$

$$
\begin{aligned}
-2 h(F)+w(F)+c_{1}(M) & =-2 n h\left(F^{\prime}\right)+d h\left(F^{\prime}\right)+w\left(F^{\prime}\right)+c_{1}(M) \\
& =(2 \varrho-2) h\left(F^{\prime}\right)+w\left(F^{\prime}\right)+c_{1}(M) .
\end{aligned}
$$

Ist speziell $w\left(F^{\prime}\right)=0$, so folgt aus $1.2,(10 \mathrm{a})$ und wegen $h\left(F^{\prime}\right) \circ h\left(F^{\prime}\right)=0$ für die Indexsumme $c_{f}$ des Feldes $f$ :

$$
c_{f}=(2 \varrho-2) h\left(F^{\prime}\right) c_{1}(M)+c_{2}(M) .
$$

Wenn $\pi$ das Geschlecht einer $H_{a}$ ohne Singularitäten ist, und alle anderen $H_{a}$ genau einen Doppelpunkt haben, also vom Geschlecht $\pi-1$ sind, und $\delta$ die Anzahl der $H_{a}$ mit Doppelpunkt ist, dann folgt weiter (vgl. 2. 2, (20a))

$$
h\left(F^{\prime}\right) \circ c_{1}(M)=2-2 \pi
$$

und

$$
\delta=-(2 \varrho-2)(2 \pi-2)+c_{2}(M)
$$


Für eine algebraische Fläche ist diese Formel bekannt ([19], p. 43):

$$
I=\delta+4(\varrho-1)(\pi-1)-4 \text {. }
$$

(Formel von Castelnuovo-Enriques).

3. 6. In diesem Abschnitt soll ein Beweis für, 2. 7. angedeutet werden. Auf die Einzelheiten des Beweises wird nicht eingegangen.

3. 6. 1. Die meromorphen Funktionen $F_{1}, F_{2}$ bestimmen (siehe 3. 1.) in $M$ Felder $f_{1}, f_{2}$ von komplexen Linienelementen. Der Ort der Punkte $P$ von $M$ mit $f_{1}(P)=f_{2}(P)$ ist eine in $M$ liegende kompakte analytische Fläche, die durch die singulären Punkte von $f_{1}, f_{2}$ hindurchgeht.

Diese analytische Fläche, deren irreduzible Komponente in natürlicher Weise mit positiven Vielfachheiten zu versehen sind, repräsentiert eine Homologieklasse $c^{\prime}$ von $M$.

Man kann zeigen (vgl. das Lemma in [12]):

$$
\omega\left(f_{1}, f_{2}\right) \text { dual zu } c^{\prime} \text {. }
$$

$h_{1}, h_{2}$ seien die Homologieklassen der Niveauflächen von $F_{1}, F_{2}$, ferner $w_{1}, w_{2}$ die Verzweigungen von $F_{1}, F_{2}$ (vgl. 1. 3).

Dann läßt sich zeigen: Der Zyklus $C$ (siehe 2. 7) gehört zur Homologieklasse

$$
-2 h_{1}+w_{1}-2 h_{2}+w_{2}+c^{\prime} \text {. }
$$

Diese Homologieklasse ist nach 3.2 und (26) dual zu

$$
\alpha\left(s, f_{1}\right)+\alpha\left(s, f_{2}\right)+\omega\left(f_{1}, f_{2}\right)-2 \gamma_{1}(M) .
$$

Aus 1. 2, (9a) und (11) folgt schließlich, daß diese Summe gleich $-\gamma_{1}(M)$ ist. Damit ist dann die Behauptung von 2. 7 bewiesen.

3. 6. 2. Es werde noch ein Beispiel für (26) angegeben.

In der komplex-projektiven Ebene seien zwei lineare Kurvenbüschel ${ }^{7}$ ) von Kurven der Ordnung $n$ bzw. $n^{\prime}$ gegeben. Der Ort der Punkte, in denen sich zwei Kurven der beiden Büschel berühren, ist eine K.urve der Ordnung $2\left(n+n^{\prime}\right)-3$ (siehe z. B. Pascal, Repertorium der höheren Mathematik II 1 (2. Auflage 1910). p. 339).

$\mathrm{Zu}$ den Kurvenbüscheln gehören die tangentialen Felder $f, f^{\prime}$ von komplexen Linienelementen. Es ist

$$
\omega\left(f, f^{\prime}\right) \text { dual zu }\left(2\left(n+n^{\prime}\right)-3\right) \cdot \text { projektive Gerade. }
$$

\section{Literatur.}

1. Behnke, $H$. und Stein, $K$., Modifikation komplexer Mannigfaltigkeiten und Riemannscher Gebiete, Math. Annalen 124, 1-16 (1951).

2. Cairns, S. S., On the triangulation of regular loci, Annals of Mathematics (2) 35, 579-587 (1934).

3. Cairns, S. S., Triangulation of the manifold of class one, Bull. Amer. Math. Soc. 41, 549-552 (1935).

4. Chern, S. S., Characteristic classes of Hermitian manifolds, Annals of Mathematics (2) 47, 85-121 (1946).

5. Ehresmann, Ch., Sur la théorie des espaces fibrés (Topologie algébrique, Paris 1947). Colloques internat. centre nat. rech. sci. Nr. 12, 3-15 (1949).

6. Hirsch, G., L'anneau de cohomologie d'un espace fibré et les classes caractéristiques, C. R. Acad. Sci. Paris 229, 1297-1299 (1949).

7. Hirsch, G., Quelques relations entre l'homologie dans les espaces fibrés et les classes caractéristiques relatives à un groupe de structure. Centre Belge rech. math., Coll. Top., 123-136 (1951).

8. Hirzebruch, $F$., Ubber eine Klasse von einfach-zusammenhängenden komplexen Mannigfaltigkeiten, Math. An nalen 124, 77-86 (1951).

9. Hirzebruch, $F$., Ubber vierdimensionale Riemannsche Flächen mehrdeutiger analytischer Funktionen von zwe $\mathrm{i}$ komplexen Veränderlichen, Dissertation Münster 1950. 
10. Hodge, W. V. D., The characteristic classes on algebraic varieties, Proc. London Math. Soc. (3) 1, 138-151 (1951).

11. Hopf, H., Zur Topologie der komplexen Mannigfaltigkeiten. Studies and Essays presented to R. Courant, New York, 167-185 (1948).

12. Hopf, $H$., Sur les champs d'éléments de surface dans les variétés à 4 dimensions. Colloques internat. centre nat. rech. sci. Nr. 12 (Topologie algébrique, Paris 1947), 55-59 (1949).

13. Hopf, H., Sur une formule de la théorie des espaces fibrés. Centre Belge rech. math., Coll. Top., 117-121 (1951).

14. Hopf, H., Utber komplex-anaiytische Mannigfaltigkeiten, Rend. Mat. e Appl. V, 10, 169-182 (1951).

15. Kundert, E. G., Über Schnittflächen in speziellen Faserungen und Felder reeller und komplexer Linienelemente, Annals of Mathematics (2) 54, 215-246 (1951).

16. Seifert, H., Topologie 3-dimensionaler gefaserter Räume, Acta Math. 60, 147-238 (1932).

17. Steenrod, $N$., The topology of fibre bundles, Princeton Mathematical Series No. 14 (1951).

18. van der Waerden, B. L., Einführung in die algebraische Geometrie. Berlin, Julius Springer (1939).

19. Zariski, O., Algebraic Surfaces. Ergebnisse der Mathematik und ihrer Grenzgebiete. Berlin, Julius Springer (1935).

Eingegangen 29. März 1952.

\section{Zusätze bei der Korrektur.}

Zusatz 1. Die Herren S. S. Chern und K. Kodaira haben mir gesagt, daß die adjunction formula aus der Dualitätsformel für die Chernschen Klassen erhalten werden kann. Für einen Beweis der Dualitätsformel siehe eine demnächst erscheinende Arbeit von S. S. Chern. Diejenigen Ergebnisse von Kundert, die in meiner Arbeit benutzt werden, können mit Hilfe der Dualitätsformel bewiesen werden. Vgl. dazu die Arbeiten von W. T. Wu. - Zu Satz I vgl. ferner: K. Kodaira, The theorem of Riemann-Roch on compact analytic surfaces, Amer. Journal of Math. 73, 813-875 (1951), insb. p. 852, (5. 4.), und Annals of Math. 56, 288-342 (1952), insb. p. 319-320 und Fußnote 45.

Zusatz 2. In einer Note in Proc. of the Nat. Acad. Sciences 38, 893-895 (1952) hat E. G. Kundert einen Satz über meromorphe Differentialformen angegeben, der den Satz II bei richtiger Interpretation von ,ppolar cycle" teilweise enthält (Anwendung des Kundertschen Theorems I auf die meromorphe Differentialform $\mathrm{d} F$ ). 